Cytologia Focus:

\title{
Synthetic Carbon Fixation: Conversion of Heterotrophs into Autotrophs by Calvin-Benson-Bassham Cycle Induction
}

\author{
Yuki Kawanishi ${ }^{1}$ and Sachihiro Matsunaga ${ }^{2 *}$ \\ ${ }^{1}$ Department of Applied Biological Science, Faculty of Science and Technology, Tokyo University of Science, \\ 2641 Yamazaki, Noda, Chiba 278-8510, Japan \\ ${ }^{2}$ Laboratory of Integrated Biology, Department of Integrated Biosciences, Graduate School of Frontier Sciences, \\ 5-1-5 Kashiwanoha, Kashiwa, Chiba 277-8561, Japan
}

Received September 25, 2021; accepted October 6, 2021

\begin{abstract}
Summary Synthetic biology has enabled us to artificially convert heterotrophs into autotrophs. Modification of the existing metabolic circuit and induction of enzymes of the Calvin-Benson-Bassham (CBB) cycle including phosphoribulokinase (PRK) and ribulose-1,5-bisphosphate carboxylase/oxygenase (RuBisCO) allowed Escherichia coli and Pichia pastoris to fix $\mathrm{CO}_{2}$. This heterotroph to autotroph conversion technology will contribute to reductions in energy consumption and $\mathrm{CO}_{2}$ emissions in the bioindustry.
\end{abstract}

Keywords Autotrophs, Heterologous, CBB cycle, RuBisCO, PRK, Synthetic biology.

Autotrophs can produce organic compounds from inorganic compounds by carbon fixation. Heterotrophs, however, cannot produce carbon from inorganic compounds, so they must eat organic compounds produced by autotrophs to live. Humans, as consumers of large amounts of fuel and food, are also directly or indirectly dependent on autotrophs. Since the industrial revolution, the burning of fossil fuels and deforestation have caused a significant increase in the concentration of $\mathrm{CO}_{2}$, one of the greenhouse gases, which has become a major social problem (Kumar et al. 2018, Irfan et al. 2019, Hosokawa and Kawano 2020). Originally, the balance of the $\mathrm{CO}_{2}$ concentration in the atmosphere was maintained through $\mathrm{CO}_{2}$ fixation by autotrophs, but our consumption activities have caused excessive $\mathrm{CO}_{2}$ emissions. To achieve a sustainable society, we need to minimize $\mathrm{CO}_{2}$ emissions and increase $\mathrm{CO}_{2}$ fixation (Ort et al. 2015). One important approach is to better understand the principles of carbon fixation in autotrophs (Smith and Stitt 2007) and how to enhance it (Kromdijk et al. 2016, Schwander et al. 2016, South et al. 2019). Building synthetic autotrophs is a good way to understand autotrophs because we can learn the factors required for metabolic pathways and the various constraints on natural autotrophs. Currently, heterotrophic organisms are mainly used in biological industrial production because autotrophs are unsuitable due to their slow growth rate and

\footnotetext{
*Corresponding author, e-mail: sachi@edu.k.u-tokyo.ac.jp DOI: $10.1508 /$ cytologia. 86.277
}

reaction efficiency; however, if autotrophs become better understood and these factors are improved, industrial heterotrophs could be replaced by autotrophs. This will help to solve social problems, including $\mathrm{CO}_{2}$ emissions. Thus, researchers in various fields such as cell engineering, molecular biology, and synthetic biology have been working towards this goal.

There are six known carbon fixation pathways in autotrophs (Berg 2011, Bar-Even et al. 2012). In particular, the Calvin-Benson-Bassham (CBB) cycle is important because it is widely distributed in most autotrophs, including algae, cyanobacteria, and plants (Liang et al. 2020). The cycle has three phases-carboxylation, a reduction reaction, and ribulose 1,5-bisphosphate (RuBP) regeneration-and is composed of 13 enzymes (Bassham et al. 1954). A key enzyme in the CBB cycle, ribulose-1,5-bisphosphate carboxylase/oxygenase (RuBisCO), fixes more than 10 tons of $\mathrm{CO}_{2}$ from the atmosphere per year (Field et al. 1998, Hayer-Hartl and Hartk 2020). RuBisCO is known to have four forms, of which forms I, II, and III are enzymes that catalyze RuBP-dependent carboxylation (Tabita 1999, Tabita et al. 2007). Eukaryotic and prokaryotic RuBisCO enzymes are commonly grouped into form I or form II (Whitney et al. 2011). RuBisCO is a very inefficient enzyme, with a very slow carboxylation turnover rate of 1-10 reactions per second and a low affinity for $\mathrm{CO}_{2}$ (Tcherkez et al. 2006). This means large amounts of $\mathrm{RuBisCO}$ are required to maintain the balance of photosynthesis versus metabolism, making RuBisCO the 
most abundant enzyme on earth (Ellis 1979, Feller et al. 2008). In addition, competition between carboxylation and oxygenation is rate-limiting, and the byproduct 2-phosphoglycolate is toxic to cells and must be metabolized in the photorespiratory cycle (Hagemann and Bauwe 2016). Therefore, many researchers have attempted to improve the carboxylation rate and $\mathrm{CO}_{2}$ affinity by molecular modification and protein-directed evolution, but unfortunately, few improvements have been achieved so far (Ducat and Silver 2012, Lin et al. 2014, Kubis and Bar-Even 2019). There are several reasons for this lack of progress, such as the complexity of the RuBisCO protein structure, the obscurity of the genetic background of autotrophs, and the absence of genetic engineering tools. Therefore, some researchers have attempted to artificially design not only RuBisCO but also the CBB cycle (Liang et al. 2020). The restructuring of RuBisCO and the rewiring of metabolic pathways in heterotrophs is a critical path to understanding autotrophs and achieving sustainability.

There are several issues involved in converting heterotrophs into autotrophs. First, chaperones and auxiliary factors are required for the folding, assembly, and functional maintenance of RuBisCO, and we still need to identify them (Bracher et al. 2017, Hayer-Hartl and Hartl 2020). Form I RuBisCO is difficult to construct in heterologous organisms because it requires several different chaperones and cofactors, but form II RuBisCO can be constructed in E. coli with only GroEL, a homo$\log$ of chloroplast chaperonin Cpn60, and its cofactor, GroES (Aigner et al. 2017, Hayer-Hartl 2017). Second, the constructed CBB cycle needs to be connected to the central carbon metabolism pathway so that all biomass is produced using carbon fixation. In autotrophs, glycerate 3-phosphate generated by $\mathrm{RuBisCO}$ is regenerated into ribulose 1,5-bisphosphate in the $\mathrm{CBB}$ cycle and simultaneously assimilated into the host to sustain life. Third, in artificial autotrophs, reducing power is deficient due to the absence of photosystems such as chloroplasts. Therefore, approaches using non-chemical energy or carbon sources that are not assimilated by the host are required. Finally, the metabolic kinetics at the branching point between the CBB cycle and the glycolytic pathway need to be optimized to maintain a stable metabolism in vivo. The optimal model of the carbon metabolism network is simulated when the CBB cycle, the glycolytic pathway, and the tricarboxylic acid cycle are connected in E. coli (Barenholz et al. 2017).

Previously, attempts have been made to convert heterotrophic model organisms, such as Escherichia coli, Methylobacterium extorquens, Saccharomyces cerevisiae, and Pichia pastoris, into autotrophs using the CBB cycle. Here, we introduce these attempts in each species. E. coli is the most widely used host organism in all biotechnology industries (Marisch et al. 2013). To select RuBisCO with higher activity in E. coli, strategies to express CBB-related genes and screening tools for directed evolution were developed (Parikh et al. 2006, Mueller-Cajar et al. 2007, Mueller-Cajar and Whitney 2008, Cai et al. 2014, Wilson et al. 2016, Zhou and Whitney 2019). When genes for RuBisCO and phosphoribulokinase (PrkA) were overexpressed in E. coli, $\mathrm{CO}_{2}$ emissions were reduced (Zhuang and Li 2013). This research showed that wild-type E. coli emits $0.731 \mathrm{~mol}$ of $\mathrm{CO}_{2}$ per mol of arabinose consumed, while engineered E. coli emits only $0.621 \mathrm{~mol}$ of $\mathrm{CO}_{2}$ per mol of arabinose. This represents a $15 \%$ reduction in $\mathrm{CO}_{2}$ emissions. In addition, Adaptive Laboratory Evolution (ALE) was developed, which uses chemostats to limit the organic carbon source and perform gradual selection (Sonderegger and Sauer 2003, Blount et al. 2012). Consequently, hemiautotrophic growth of $E$. coli was enabled using ALE (Antonovsky et al. 2016). In that study, to establish the CBB cycle, form II RuBisCO from Rhodospirillum rubrum and prkA from Synechococcus elongatus were expressed and evolved in xylose-limited chemostats. The result was an E. coli strain that produced a third of its biomass from $\mathrm{CO}_{2}$ and required pyruvate for reducing power and as an energy source for stable growth. In a subsequent study, a strain of $E$. coli that was a fully autotrophic was established by expressing formate dehydrogenase (FDH) from methylotrophic bacteria, knocking out several genes in the glycolytic system, and evolving E. coli with chemostats (Gleizer et al. 2019) (Fig. 1). Formate is oxidized by FDH, which is the reducing force of the CBB cycle. Sequencing of these E. coli clones revealed mutations in genes involved in the branching of the $\mathrm{CBB}$ cycle or in the promoter region that controls NADH production, which suggested that a proper balance is required to maintain stable metabolism.

Yeast is commonly used in the large-scale production of bioethanol as an alternative to fossil fuels because of its strong ethanol tolerance (Alper et al. 2006, Della-Bianca et al. 2013, Nielsen et al. 2013). It is also an important host for the industrial production of biopharmaceuticals and useful proteins (Li et al. 2015, Xue et al. 2017, Duan et al. 2018). In the yeast S. cerevisiae, RuBisCO and PRK from various origins were investigated and introduced to reduce $\mathrm{CO}_{2}$ emissions during bioethanol fermentation (Guadalupe-Medina et al. 2013, Xia et al. 2017, Papapetridis et al. 2018). As a result, the CBB cycle, which reoxidizes NADH using $\mathrm{CO}_{2}$ as an electron acceptor, showed improved ethanol yield, decreased byproducts, and reduced $\mathrm{CO}_{2}$ emissions. Notably, form I was demonstrated to have higher carboxylation activity than form II when form I RuBisCO from Ralstonia eutropha and form II RuBisCO from Thiobacillus denitrificans were each heterologously expressed in $S$. cerevisiae (Li et al. 2017). In addition, an engineered strain of $M$. extorquens AM1 was designed by implementing the CBB cycle and knocking out the host genes, but this strain was unable to grow continuously using 

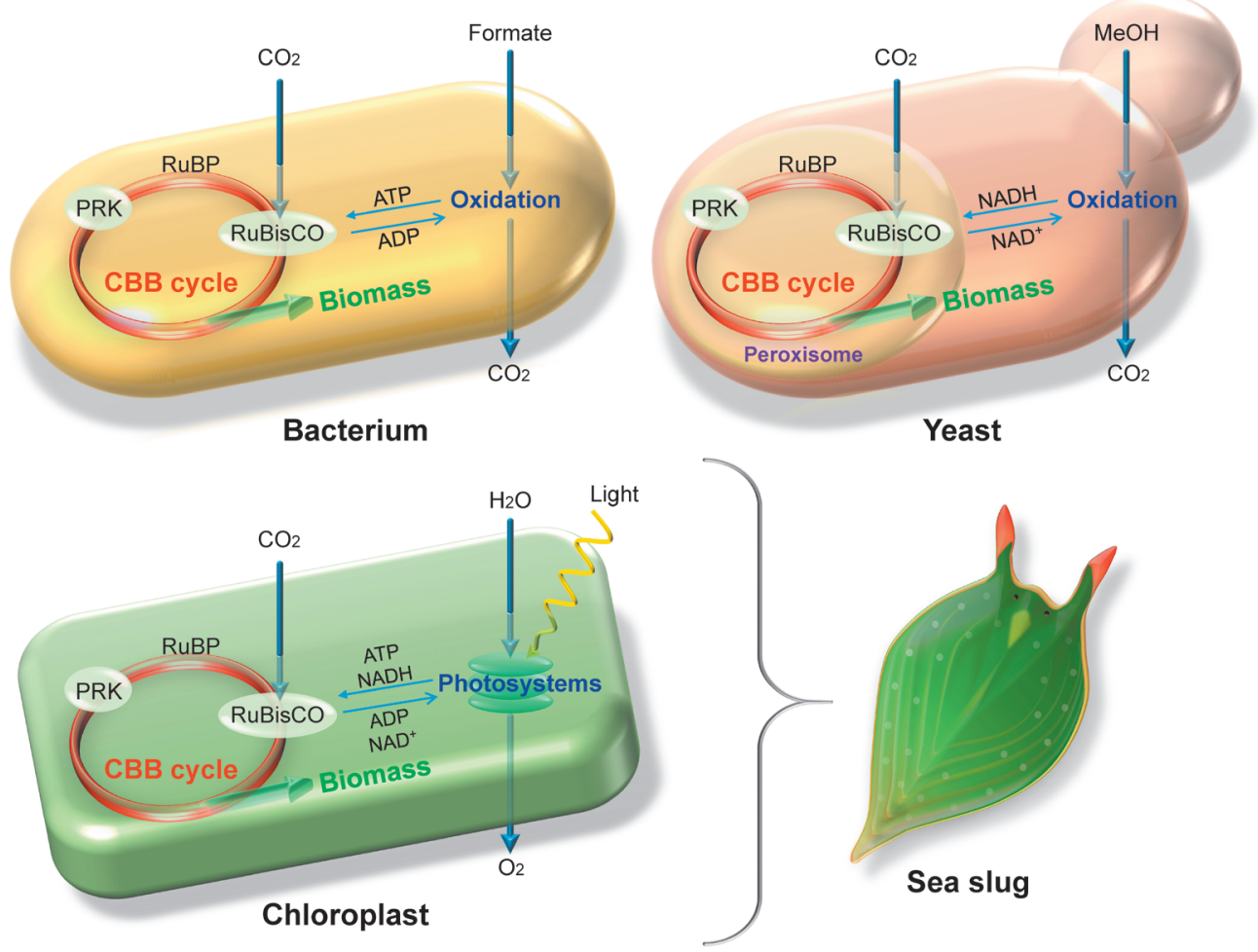

Fig. 1. Metabolic models of autotrophs living on $\mathrm{CO}_{2}$ as a sole carbon source. The engineered bacterium (Escherichia coli) obtained a biomass production pathway through carbon fixation in the CBB cycle and an energy production pathway through oxidation of formate. These metabolic pathways were optimized using chemostats. The engineered yeast (Pichia pastoris) acquired a peroxisome-localized CBB cycle and energy production pathway through methanol assimilation. Sea slugs (Plakobranchus ocellatus) in nature can sequester functional chloroplasts from algae into their cells via kleptoplasty. This enables them to produce biomass through the photosystems and the CBB cycle in chloroplasts.

$\mathrm{CO}_{2}$ as a sole carbon source (Schada von Borzyskowski et al. 2018). More recently, PRK and RuBisCO were introduced into P. pastoris and two genes of dihydroxyacetone synthase and a gene of alcohol oxidase in the assimilation pathway were knocked out, resulting in the establishment of a fully autotrophic strain that can grow continuously with $\mathrm{CO}_{2}$ as a sole carbon source and methanol as an energy source (Gassler et al. 2020) (Fig. 1). Surprisingly, the growth rate of this strain was improved to the same level as the wild type using chemostats. This suggested that this strain has the potential to become a basic organism for industrial production and contribute to the reduction of $\mathrm{CO}_{2}$ emissions.

Finally, although there are no reports of converting animal cultured cells into autotrophs, we introduce some reports that may support the idea. In nature, a variety of photosynthetic animals evolved through secondary symbiosis with algae (Rumpho et al. 2011, Van Steenkiste et al. 2019). In particular, sea slugs can sequester functional chloroplasts from algae into their cells via a symbiotic phenomenon called kleptoplasty, which allows them to live solely on photosynthetic products like autotrophs (Yamamoto et al. 2013, Laetz and Wägele 2017, Aoki and Matsunaga 2021) (Fig. 1). For a long time, researchers have discussed whether horizontal transfer of algal genes may have occurred in sea slugs to maintain chloroplasts without algal nuclei during evolution, but surprisingly, a recent study showed that horizontal transfer has not occurred in the kleptoplastic sea slug Plakobranchus ocellatus (Maeda et al. 2021). This implies that complex biological machinery such as chloroplasts can be transferred across species; in other words, an animal can also acquire the ability to photosynthesize.

These achievements in synthetic carbon fixation will accelerate the construction of artificial photosynthetic animal cells (Matsunaga 2018, Puri et al. 2021). Although there are still open issues to address in the conversion of heterotrophs into autotrophs, further development will reveal universal principles for conversion. Conversion technology based on these principles will contribute to finding an effective solution to reduce humanity's huge energy consumption and carbon dioxide emissions, leading to global environmental improvement.

\section{Acknowledgements}

This research was supported by grants from MXT/ JSPS KAKENHI (20H05911) and JST, CREST Grant Number JPMJCR20S6 to SM. We thank Robbie Lewis, MSc, from Edanz (https://jp.edanz.com/ac) for editing a draft of this manuscript. 


\section{References}

Aigner, H., Wilson, R. H., Bracher, A., Calisse, L., Bhat, J. Y., Hartl, F. U. and Hayer-Hartl, M. 2017. Plant RuBisCo assembly in E. coli with five chloroplast chaperones including BSD2. Science 358: $1272-1278$.

Alper, H., Moxley, J., Nevoigt, E., Fink, G. R. and Stephanopoulos, G. 2006. Engineering yeast transcription machinery for improved ethanol tolerance and production. Science 314: 1565-1568.

Antonovsky, N., Gleizer, S., Noor, E., Zohar, Y., Herz, E., Barenholz, U., Zelcbuch, L., Amram, S., Wides, A., Tepper, N., Davidi, D., Bar-On, Y., Bareia, T., Wernick, D. G., Shani, I., Malitsky, S., Jona, G., Bar-Even, A. and Milo, R. 2016. Sugar synthesis from $\mathrm{CO}_{2}$ in Escherichia coli. Cell 166: 115-125.

Aoki, R. and Matsunaga, S. 2021. A photosynthetic animal: A Sacoglossan sea slug that steals chloroplasts. Cytologia 86: 103-107.

Barenholz, U., Davidi, D., Reznik, E., Bar-On, Y., Antonovsky, N., Noor, E. and Milo, R. 2017. Design principles of autocatalytic cycles constrain enzyme kinetics and force low substrate saturation at flux branch points. eLife 6: e20667.

Bar-Even, A., Noor, E. and Milo, R. 2012. A survey of carbon fixation pathways through a quantitative lens. J. Exp. Bot. 63: 2325-2342.

Bassham, J. A., Benson, A. A., Kay, L. D., Harris, A. Z., Wilson, A. T. and Calvin, M. 1954. The path of carbon in photosynthesis. XXI. The cyclic regeneration of carbon dioxide acceptor1. J. Am. Chem. Soc. 76: 1760-1770.

Berg, I. A. 2011. Ecological aspects of the distribution of different autotrophic $\mathrm{CO}_{2}$ fixation pathways. Appl. Environ. Microbiol. 77: 1925-1936.

Blount, Z. D., Barrick, J. E., Davidson, C. J. and Lenski, R. E. 2012. Genomic analysis of a key innovation in an experimental Escherichia coli population. Nature 489: 513-518.

Bracher, A., Whitney, S. M., Hartl, F. U. and Hayer-Hartl, M. 2017. Biogenesis and metabolic maintenance of Rubisco. Annu. Rev. Plant Biol. 68: 29-60.

Cai, Z., Liu, G., Zhang, J. and Li, Y. 2014. Development of an activity-directed selection system enabled significant improvement of the carboxylation efficiency of Rubisco. Protein Cell 5: 552-562.

Della-Bianca, B. E., Basso, T. O., Stambuk, B. U., Basso, L. C. and Gombert, A. K. 2013. What do we know about the yeast strains from the Brazilian fuel ethanol industry? Appl. Microbiol. Biotechnol. 97: 979-991.

Duan, X., Gao, J. and Zhou, Y. J. 2018. Advances in engineering methylotrophic yeast for biosynthesis of valuable chemicals from methanol. Chin. Chem. Lett. 29: 681-686.

Ducat, D. C. and Silver, P. A. 2012. Improving carbon fixation pathways. Curr. Opin. Chem. Biol. 16: 337-344.

Ellis, R. J. 1979. The most abundant protein in the world. Trends Biochem. Sci. 4: 241-244

Feller, U., Anders, I. and Mae, T. 2008. Rubiscolytics: Fate of Rubisco after its enzymatic function in a cell is terminated. J. Exp. Bot. 59: 1615-1624.

Field, C. B., Behrenfeld, M. J., Randerson, J. T. and Falkowski, P. 1998. Primary production of the piosphere: Integrating terrestrial and oceanic components. Science 281: 237-240.

Gassler, T., Sauer, M., Gasser, B., Egermeier, M., Troyer, C., Causon, T., Hann, S., Mattanovich, D. and Steiger, M. G. 2020. The industrial yeast Pichia pastoris is converted from a heterotroph into an autotroph capable of growth on $\mathrm{CO}_{2}$. Nat. Biotechnol. 38: 210-216.

Gleizer, S., Ben-Nissan, R., Bar-On, Y. M., Antonovsky, N., Noor, E., Zohar, Y., Jona, G., Krieger, E., Shamshoum, M., Bar-Even, A. and Milo, R. 2019. Conversion of Escherichia coli to generate all biomass carbon from $\mathrm{CO}_{2}$. Cell 179: 1255-1263.e12.

Guadalupe-Medina, V., Wisselink, H. W., Luttik, M. A., de Hulster, E., Daran, J.-M., Pronk, J. T. and van Maris, A. J. 2013. Carbon dioxide fixation by Calvin-Cycle enzymes improves ethanol yield in yeast. Biotechnol. Biofuels 6: 125 .

Hagemann, M. and Bauwe, H. 2016. Photorespiration and the potential to improve photosynthesis. Curr. Opin. Chem. Biol. 35: 109-116.

Hayer-Hartl, M. 2017. From chaperonins to Rubisco assembly and metabolic repair. Protein Sci. 26: 2324-2333.

Hayer-Hartl, M. and Hartl, F. U. 2020. Chaperone machineries of Rubisco-The most abundant enzyme. Trends Biochem. Sci. 45: 748-763.

Hosokawa, S. and Kawano, S. 2020. Worldwide research trends on microalgae and recent work in Cytologia. Cytologia 85: 179-187.

Irfan, M., Bai, Y., Zhou, L., Kazmi, M., Yuan, S., Maurice Mbadinga, S., Yang, S.-Z., Liu, J. F., Sand, W., Gu, J.-D. and Mu, B.-Z. 2019. Direct microbial transformation of carbon dioxide to value-added chemicals: A comprehensive analysis and application potentials. Bioresour. Technol. 288: 121401.

Kromdijk, J., Głowacka, K., Leonelli, L., Gabilly, S. T., Iwai, M., Niyogi, K. K. and Long, S. P. 2016. Improving photosynthesis and crop productivity by accelerating recovery from photoprotection. Science 354: 857-861.

Kubis, A. and Bar-Even, A. 2019. Synthetic biology approaches for improving photosynthesis. J. Exp. Bot. 70: 1425-1433.

Kumar, M., Sundaram, S., Gnansounou, E., Larroche, C. and Thakur, I. S. 2018. Carbon dioxide capture, storage and production of biofuel and biomaterials by bacteria: A review. Bioresour. Technol. 247: 1059-1068.

Laetz, E. M. J. and Wägele, H. 2017. Chloroplast digestion and the development of functional kleptoplasty in juvenile Elysia timida (Risso, 1818) as compared to short-term and non-chloroplastretaining sacoglossan slugs. PLoS One 12: e0182910.

Li, P., Sun, H., Chen, Z., Li, Y. and Zhu, T. 2015. Construction of efficient xylose utilizing Pichia pastoris for industrial enzymeproduction. Microb. Cell Fact. 14: 22.

Li, Y.-J., Wang, M.-M., Chen, Y.-W., Wang, M., Fan, L.-H. and Tan, T.-W. 2017. Engineered yeast with a $\mathrm{CO}_{2}$-fixation pathway to improve the bio-ethanol production from xylose-mixed sugars. Sci. Rep. 7: 43875.

Liang, B., Zhao, Y. and Yang, J. 2020. Recent advances in developing artificial autotrophic microorganism for reinforcing $\mathrm{CO}_{2}$ Fixation. Front. Microbiol. 11: 2848.

Lin, M. T., Occhialini, A., Andralojc, P. J., Parry, M. A. J. and Hanson, M. R. 2014. A faster Rubisco with potential to increase photosynthesis in crops. Nature 513: 547-550.

Maeda, T., Takahashi, S., Yoshida, T., Shimamura, S., Takaki, Y., Nagai, Y., Toyoda, A., Suzuki, Y., Arimoto, A., Ishii, H., Satoh, N., Nishiyama, T., Hasebe, M., Maruyama, T., Minagawa, J., Obokata, J. and Shigenobu, S. 2021. Chloroplast acquisition without the gene transfer in kleptoplastic sea slugs, Plakobranchus ocellatus. eLife 10: e60176.

Marisch, K., Bayer, K., Cserjan-Puschmann, M., Luchner, M. and Striedner, G. 2013. Evaluation of three industrial Escherichia coli strains in fed-batch cultivations during high-level SOD protein production. Microb. Cell Fact. 12: 58.

Matsunaga, S. 2018. Planimal cells: Artificial photosynthetic animal cells inspired by endosymbiosis and photosynthetic animals. Cytologia 83: 3-6.

Mueller-Cajar, O., Morell, M. and Whitney, S. M. 2007. Directed evolution of Rubisco in Escherichia coli reveals a specificitydetermining hydrogen bond in the form II enzyme. Biochemistry 46: $14067-14074$.

Mueller-Cajar, O. and Whitney, S. M. 2008. Evolving improved Synechococcus Rubisco functional expression in Escherichia coli. Biochem. J. 414: 205-214.

Nielsen, J., Larsson, C., van Maris, A. and Pronk, J. 2013. Metabolic engineering of yeast for production of fuels and chemicals. Curr. 
Opin. Biotechnol. 24: 398-404.

Ort, D. R. et al. 2015. Redesigning photosynthesis to sustainably meet global food and bioenergy demand. Proc. Natl. Acad. Sci. U.S.A. 112: 8529-8536.

Papapetridis, I., Goudriaan, M., Vázquez Vitali, M., de Keijzer, N. A., van den Broek, M., van Maris, A. J. A. and Pronk, J. T. 2018. Optimizing anaerobic growth rate and fermentation kinetics in Saccharomyces cerevisiae strains expressing Calvin-cycle enzymes for improved ethanol yield. Biotechnol. Biofuels 11: 17.

Parikh, M. R., Greene, D. N., Woods, K. K. and Matsumura, I. 2006. Directed evolution of RuBisCO hypermorphs through genetic selection in engineered E. coli. Protein Eng. Des. Sel. 19: 113-119.

Puri, K. M., Butardo, V. Jr. and Sumer, H. 2021. Evaluation of natural endosymbiosis for progress towards artificial endosymbiosis. Symbiosis 84: 1-17.

Rumpho, M. E., Pelletreau, K. N., Moustafa, A. and Bhattacharya, D. 2011. The making of a photosynthetic animal. J. Exp. Biol. 214: 303-311.

Schada von Borzyskowski, L., Carrillo, M., Leupold, S., Glatter, T., Kiefer, P., Weishaupt, R., Heinemann, M. and Erb, T. J. 2018. An engineered Calvin-Benson-Bassham cycle for carbon dioxide fixation in Methylobacterium extorquens AM1. Metab. Eng. 47: 423-433.

Schwander, T., Schada von Borzyskowski, L., Burgener, S., Cortina, N. S. and Erb, T. J. 2016. A synthetic pathway for the fixation of carbon dioxide in vitro. Science 354: 900-904.

Smith, A. M. and Stitt, M. 2007. Coordination of carbon supply and plant growth. Plant Cell Environ. 30: 1126-1149.

Sonderegger, M. and Sauer, U. 2003. Evolutionary engineering of Saccharomyces cerevisiae for anaerobic growth on xylose. Appl. Environ. Microbiol. 69: 1990-1998.

South, P. F., Cavanagh, A. P., Liu, H. W. and Ort, D. R. 2019. Synthetic glycolate metabolism pathways stimulate crop growth and productivity in the field. Science 363: eaat 9077.

Tabita, F. R. 1999. Microbial ribulose 1,5-bisphosphate carboxylase/ oxygenase: A different perspective. Photosynth. Res. 60: 1-28.
Tabita, F. R., Hanson, T. E., Li, H., Satagopan, S., Singh, J. and Chan, S. 2007. Function, structure, and evolution of the RubisCO-like proteins and their RubisCO homologs. Microbiol. Mol. Biol. Rev. 71: $576-599$.

Tcherkez, G. G. B., Farquhar, G. D. and Andrews, T. J. 2006. Despite slow catalysis and confused substrate specificity, all ribulose bisphosphate carboxylases may be nearly perfectly optimized. Proc. Natl. Acad. Sci. U.S.A. 103: 7246-7251.

Van Steenkiste, N. W. L., Stephenson, I., Herranz, M., Husnik, F., Keeling, P. J. and Leander, B. S. 2019. A new case of kleptoplasty in animals: Marine flatworms steal functional plastids from diatoms. Sci. Adv. 5: eaaw4337.

Whitney, S. M., Houtz, R. L. and Alonso, H. 2011. Advancing our understanding and capacity to engineer nature's $\mathrm{CO}_{2}$-sequestering enzyme, Rubisco. Plant Physiol. 155: 27-35.

Wilson, R. H., Alonso, H. and Whitney, S. M. 2016. Evolving Methanococcoides burtonii archaeal Rubisco for improved photosynthesis and plant growth. Sci. Rep. 6: 22284.

Xia, P.-F., Zhang, G.-C., Walker, B., Seo, S.-O., Kwak, S., Liu, J.-J., Kim, H., Ort, D. R., Wang, S.-G. and Jin, Y.-S. 2017. Recycling carbon dioxide during xylose fermentation by engineered Saccharomyces cerevisiae. ACS Synth. Biol. 6: 276-283.

Xue, Y., Kong, C., Shen, W., Bai, C., Ren, Y., Zhou, X., Zhang, Y. and Cai, M. 2017. Methylotrophic yeast Pichia pastoris as a chassis organism for polyketide synthesis via the full citrinin biosynthetic pathway. J. Biotechnol. 242: 64-72.

Yamamoto, S., Hirano, Y. M., Hirano, Y. J., Trowbridge, C. D., Akimoto, A., Sakai, A. and Yusa, Y. 2013. Effects of photosynthesis on the survival and weight retention of two kleptoplastic sacoglossan opisthobranchs. J. Mar. Biol. Assoc. U. K. 93: 209-215.

Zhou, Y. and Whitney, S. 2019. Directed evolution of an improved Rubisco; in vitro analyses to decipher fact from fiction. Int. J. Mol. Sci. 20: 5019.

Zhuang, Z.-Y. and Li, S.-Y. 2013. Rubisco-based engineered Escherichia coli for in situ carbon dioxide recycling. Bioresour. Technol. 150: 79-88. 